\title{
Lymphom der Prostata - Diagnose auf den „zweiten Stich“
}

\author{
Gabi Vetsch ${ }^{1}$, Christa K. Baumann ${ }^{2}$, Marco Kläy ${ }^{1}$, Nicolas Leupin ${ }^{2}$, Cyrill Rentsch ${ }^{3}$, Esther Mueller-Garamvölgyi ${ }^{4}$,
} Ulrich Bürgi ${ }^{1}$, Uwe Schiemann ${ }^{1}$

\section{ZUSAMMENFASSUNG}

$\square$ Hintergrund: Maligne Lymphome der Prostata sind eine klinische Rarität. In der Literatur werden insgesamt etwa 165 Fälle beschrieben, in denen ein primäres Lymphom oder eine sekundäre Infiltration der Prostata durch ein Lymphom vorlag.

$\square$ Fallbeschreibung: Berichtet wird der Fall eines 59-jährigen Patienten mit einer unscharf begrenzten Raumforderung im Bereich der Prostata bei normalem prostataspezifischem Antigen (PSA), einer Wirbelkörperfraktur und bilateralen Nebennierenvergrößerungen. Eine zweizeitige Prostatastanzbiopsie erbrachte die Diagnose eines diffus-großzelligen B-Zell-Non-Hodgkin-Lymphoms. Weitere Staginguntersuchungen ergaben Anhaltspunkte für das Vorliegen eines epiduralen Befalls. Es wurde eine Polychemotherapie inklusive intrathekaler Applikation initiiert, unter der sich die Lymphommanifestationen bei einem ersten Zwischenstaging nach vier Zyklen deutlich zurückgebildet hatten. Nach weiteren zwei Zyklen R-CHOP zeigte ein CT zwar noch ein kleines Weichteilplus in der Prostataloge, im anschließenden PET-CT war jedoch kein vitales Lymphomgewebe mehr nachweisbar (komplette Remission).

$\square$ Schlussfolgerung: Bei einer Raumforderung im Bereich der Prostata muss neben einem Prostatakarzinom als weitaus häufigste Tumorentität auch an ein solitäres Lymphom oder eine Infiltration der Prostata bei generalisiertem Lymphom gedacht werden, insbesondere bei einem normalen PSA.

Schlüsselwörter: Prostatakarzinom · Malignes Lymphom (der Prostata)

Med Klin 2008;103:245-8.

DOI 10.1007/s00063-008-1034-y

\section{ABSTRACT}

Malignant Lymphoma of the Prostate - Diagnosis on the Second Biopsy

$\square$ Background: Malignant lymphoma of the prostate is rare. In the literature, about 165 cases with either a primary lymphoma of the prostate or secondary infiltration of the prostate by a lymphoma are described.

$\square$ Case Report: The case of a 59-year-old patient with an irregular tumor in the prostatic region, but normal prostate-specific antigen (PSA), a fracture in the vertebral column and a bilateral enlargement of the suprarenal glands is presented. Repetitive prostate biopsy revealed the diagnosis of a diffuse large

\footnotetext{
${ }^{1}$ Klinik und Poliklinik für Allgemeine Innere Medizin, Inselspital Bern, Universität Bern, Schweiz,

${ }^{2}$ Klinik für Onkologie, Inselspital Bern, Universität Bern, Schweiz,

${ }^{3}$ Urologische Klinik, Inselspital Bern, Universität Bern, Schweiz,

${ }^{4}$ Institut für Pathologie, Inselspital Bern, Universität Bern, Schweiz.

Eingang des Manuskripts: 8. 8. 2007.

Annahme des überarbeiteten Manuskripts: 30. 11. 2007.
}

\section{Fallbeschreibung}

$\square$ Anamnese und klinischer Untersuchungsbefund: Wir berichten den Fall eines 59-jährigen Patienten, der sich 3 Wochen vor Hospitalisation wegen chronischer Müdigkeit und Rückenschmerzen in hausärztliche Abklärung begeben hatte. Aus der Vorgeschichte waren degenerative Wirbelsäulenveränderungen, eine Psoriasis, Arthrosen des oberen Sprunggelenks beidseits und einige kleinere Verletzungen des Bewegungsapparats bekannt.

Hausärztlicherseits wurden ein Röntgen-Thorax in zwei Ebenen sowie eine Abdomensonographie veranlasst und eine analgetische Therapie begonnen. In einem auswärtigen Krankenhaus wurde zudem eine Computertomographie des Abdomens mit Kontrastmittel durchgeführt. Die bildgebenden Befunde ergaben den Verdacht auf ein organüberschreitendes, gegen die Blase wachsendes Prostatakarzinom, eine Deckplattenimpressionsfraktur des zweiten Lendenwirbelkörpers (LWK2, interpretiert als pathologische Fraktur bei möglicher Metastasierung) und eine bilaterale Nebennierenvergrößerung. Im Aufnahmelabor zeigte sich lediglich ein leicht erhöhtes C-reaktives Protein mit $6,2 \mathrm{mg} / \mathrm{l}$. Die übrigen Routineparameter lagen im Normbereich. Zusätzlich wurden die Tumormarker karzinoembryonales Antigen, CA 19-9, Calcitonin, Thyreoglobulin und prostataspezifisches Antigen (PSA) sowie die Nebennierenrindenhormone bestimmt, welche ebenfalls sämtlich im Normbereich lagen. Die auswärtig durchgeführte Feinnadelpunktion der Prostata erbrachte jedoch keinen Nachweis malignitätsverdächtiger Parenchymveränderungen, so dass uns der Patient mit Verdacht auf ein metastasierendes Karzinom unklaren Ursprungs und persistierenden Schmerzen 
zur weiteren Diagnostik zugewiesen wurde.

$\square$ Diagnostik: Im Rahmen der urologischen Abklärung wurde bei dem $\mathrm{Pa}$ tienten eine Zystoskopie durchgeführt. Diese zeigte endoluminal normale Schleimhautverhältnisse, jedoch eine Imprimierung der Blasenhinterwand, welche mit einem Druck von außen durch eine Prostatavergrößerung vereinbar war. In der Spülzytologie fanden sich keine malignitätsverdächtigen Zellen. Trotz erstmalig negativer Feinnadelpunktion der Prostata wurde eine zweite Biopsie entnommen, da die radiologischen und endoskopischen Befunde auch bei nochmalig kontrolliertem, normalem PSA konklusiv für einen von der Prostata ausgehenden raumfordernden Prozess waren. Postinterventionell kam es zu einer Makrohämaturie mit partieller Blasentamponade, so dass der Patient vorübergehend (für 48 h) auf die Urologie verlegt, die Blasentamponade ausgeräumt und ein Spülkatheter eingelegt wurden.

Die histologische Begutachtung inklusive Immunhistochemie der zweiten Prostatabiopsie erbrachte die abschließende Diagnose eines diffus-großzelligen B-Zell-Lymphoms nach WHO (Abbildungen 1 und 2). Das Staging wurde entsprechend um eine Compu-

Tabelle 1. Stadieneinteilung nach der Ann-Arbor-Klassifikation.

$\begin{array}{ll}\text { Stadium I } & \begin{array}{l}\text { Befall einer einzelnen Lymphknotenregion oder lokalisierter Befall } \\ \text { einer extranodalen Struktur ohne gleichzeitigen Lymphknoten- } \\ \text { befall (IE) }\end{array} \\ \text { Stadium II } & \begin{array}{l}\text { Befall mehrerer Lymphknotenregionen auf der gleichen Seite des } \\ \text { Diaphragmas oder lokalisierter Befall einer extranodalen Struktur } \\ \text { mit Befall der benachbarten Lymphknotenregionen (IIE) }\end{array} \\ \text { Stadium III } & \begin{array}{l}\text { Befall von Lymphknotenregionen auf beiden Seiten des Diaphrag- } \\ \text { mas. Zusätzlich kann die Milz (IIIS) oder lokalisiert eine extranodale } \\ \text { Struktur (IIIE) oder beide (IIISE) befallen sein. Der infradiaphrag- } \\ \text { male Anteil kann in Stadium III(1) mit Befall der portalen, zöliakalen } \\ \text { oder perisplenischen Lymphknoten oder in Stadium III(2) mit Befall } \\ \text { der paraaortalen, iliakalen und mesenterialen Lymphknoten } \\ \text { unterteilt werden }\end{array} \\ \text { Siffuser Befall eines oder mehrerer extranodaler Organe oder } \\ \text { Strukturen mit oder ohne Lymphknotenbefall }\end{array}$
vital lymphatic tissue (complete remission). ertertomographie von Hals bis Becken (Abbildungen 3 und 4), eine Magnetresonanztomographie des Schädels, eine Liquoruntersuchung und eine Knochenmarkpunktion ergänzt. Computertomographisch zeigte sich im Vergleich zur Voruntersuchung eine GröBenprogredienz der Lymphommassen mit Infiltration der Prostata, Samenblase, Harnblase sowie eines linksiliakalen und zweier rechtsretrokavaler Lymphknoten und den bekannten Raumfor-

B cell lymphoma. Further staging examinations gave hints to an epidural infiltration. A polychemotherapy including intrathecal drug applications was initiated. Staging after four therapeutic cycles already showed good partial remission of all lymphoma manifestations. After two further therapeutic cycles, a CT scan showed a small rest of prostatic bulk, but PET-CT did not detect

$\square$ Conclusion: In cases of irregular prostatic enlargements, carcinoma has to be considered as the most frequent diagnosis. Nevertheless, also a solitary lymphoma or infiltration of the prostate by a systemic lymphoma has to be taken into account, especially if the PSA value is in the normal range.

Key Words: Prostatic cancer - Malignant lymphoma (of the prostate) Med Klin 2008;103:145-8. DOI 10.1007/s00063-008-1034-y

derungen der Nebennieren beidseits; im Übrigen bestand der Verdacht einer pathologischen Deckplattenfraktur LWK2. Ebenso stellte sich eine epidurale Raumforderung mit Ausdehnung entlang den Intervertebralforamina BWK11 (Brustwirbelkörper 11) bis LWK2 dar. Die durchgeführte Liquoruntersuchung zeigte keine malignitätsverdächtigen Zellen, und das Magnetresonanztomogramm des Schädels ergab keine Hinweise auf eine Meningeosis lymphomatosa. Im Material des Knochenmarkzylinders und des Knochenmarkaspirats fanden sich keine Hinweise für eine Infiltration durch das diffus-großzellige B-Zell-Lymphom.

Diagnose: diffus-großzelliges BZell-Non-Hodgkin-Lymphom der Prostata, Stadium IVAE nach Ann-Arbor (Tabelle 1).

\section{DISKUSSION}

Das Non-Hodgkin-Lymphom (NHL) ist eine Neoplasie des lymphatischen Gewebes, ausgehend vom B-Zell(B-Zell-NHL) oder T-Zell-System (T-Zell-NHL). Je nach Histologie und klinischem Verlauf wird zwischen High-Grade- und Low-Grade-NHL unterschieden. Die WHO-Klassifikation stellt den internationalen Standard der Lymphomklassifikation dar und hat frühere Ansätze (KIEL, REAL, Working Formulation) weitgehend ersetzt [1]. Eine bestimmte Lymphomentität 


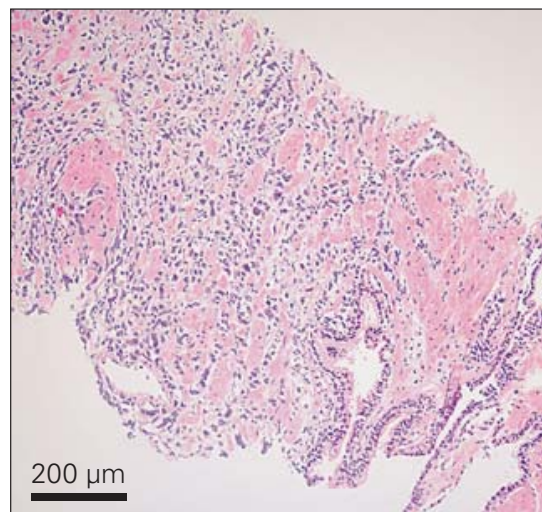

Abbildung 1. Prostatabiopsie mit Infiltration des fibromuskulären Stromas durch mittelgroße bis große lymphoide Blasten zwischen intakten Prostatadrüsen (HE-Färbung, Vergrößerung $10 \times)$.

wird aufgrund aller verfügbaren Informationen, nämlich Morphologie, Immunphänotyp, genetische Befunde sowie klinische Merkmale, diagnostiziert.

High-Grade-NHL sind charakterisiert durch einen raschen, ohne Therapie meist tödlichen Verlauf, jedoch auch durch die potentiell kurativen Therapieoptionen, sogar in fortgeschrittenen Stadien. Die Inzidenz der High-Grade-NHL hat in den letzten Jahrzehnten zugenommen. Sie machen ca. 3\% aller malignen Erkrankungen aus. Der Häufigkeitsgipfel liegt zwischen dem 40. und 80. Lebensjahr. Das großzellige B-Zell-Lymphom wird zu den High-Grade-Lymphomen gezählt,

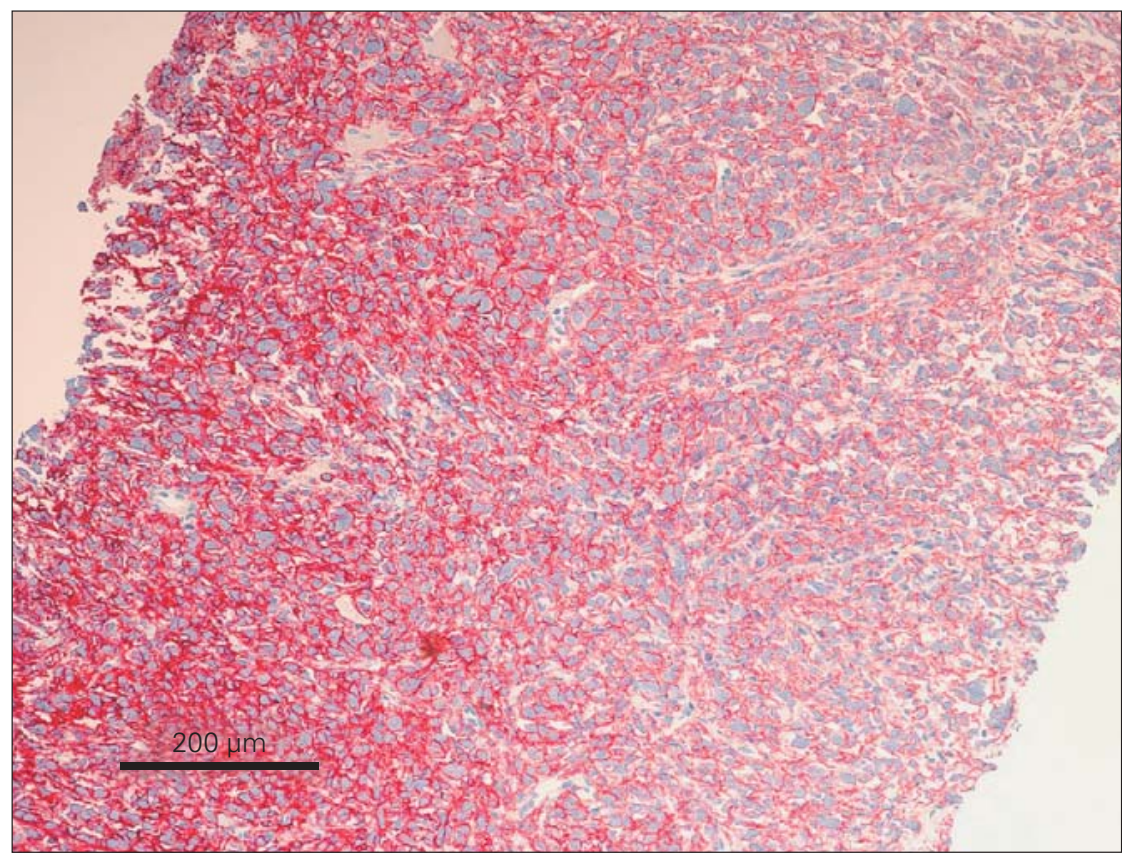

Abbildung 2. Blasten mit deutlicher Immunreaktivität für den B-Zell-Marker CD20 (immunhistochemische Färbung für CD20, Vergrößerung 10×).

es stellt eine diffuse Proliferation groBer neoplastischer lymphoider B-Zellen dar. Es bildet zudem die größte Gruppe mit einem Anteil von 30-40\% aller diagnostizierten Lymphome [2].

Das maligne Lymphom der Prostata ist eine klinische Rarität. Erstmals wurde im Jahre 1877 in der Literatur über einen Patienten mit einem Lymphom der Prostata berichtet [3]. Prostatalymphome können allein oder im Rahmen eines generalisierten Lym- phoms auftreten [4]. Insgesamt werden in der Literatur nur 165 Fälle beschrieben [5-7]. Meist wird die Diagnose eines Lymphoms der Prostata im Rahmen einer Prostatabiopsie, einer transurethralen Resektion der Prostata oder nach Prostatektomie bei Verdacht auf Prostatakarzinom gestellt [8]. Wie Bostwick et al. zeigten, handelt es sich in den meisten Fällen eines NHL der Prostata um ein Lymphom vom B-Zell-Typ [9]. Eine Infiltration der

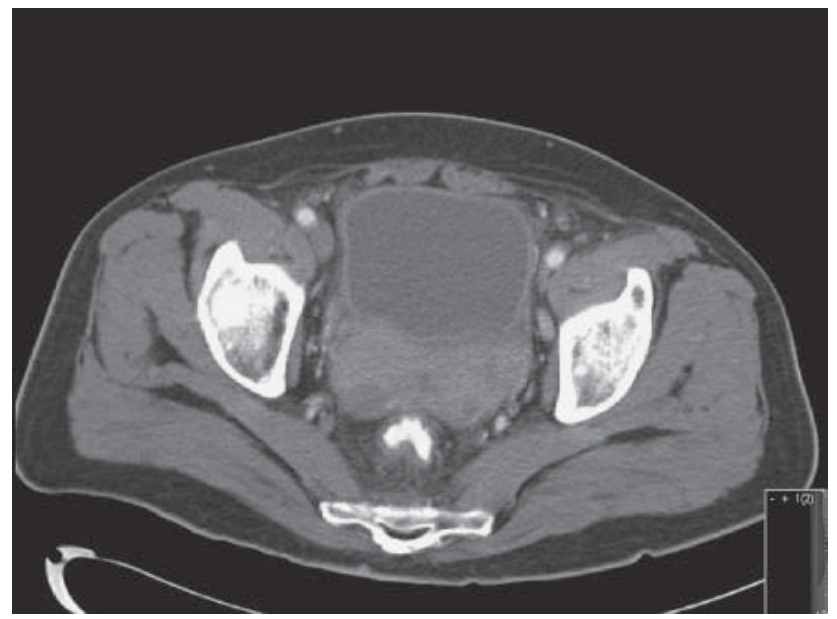

Abbildung 3. Computertomographie Abdomen/Becken: Lymphommasse mit Infiltration der Prostata, Samenblase und Harnblase im kleinen Becken.

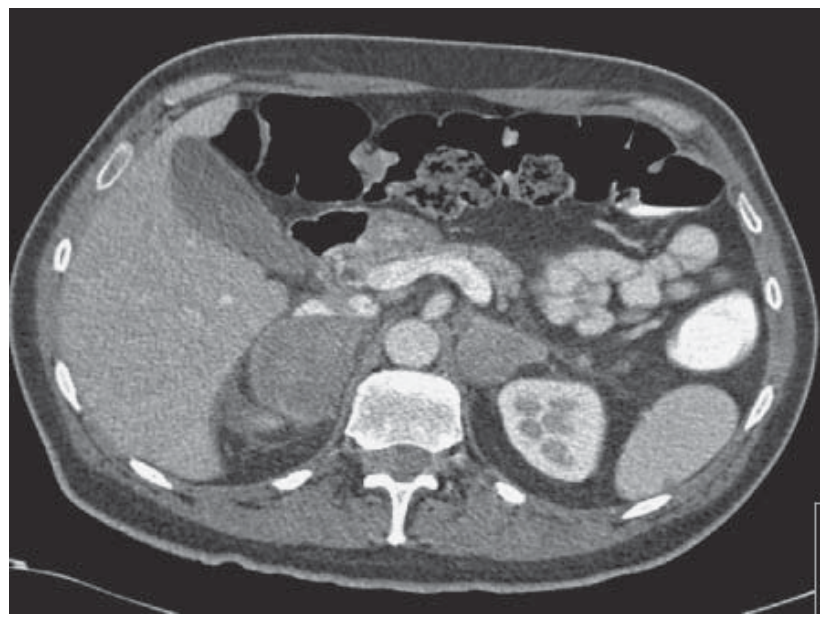

Abbildung 4. Computertomographie Abdomen/Becken: beidseitige Raumforderungen der Nebennieren. 
Prostata aufgrund eines systemischen Lymphoms ist häufiger als die alleinige Infiltration, wenn auch in der Gesamtheit aller extranodalen Lokalisationen immer noch als seltene Entität [10, 11]. Große Studien haben gezeigt, dass das diffus-großzellige B-Zell-Lymphom in bis $\mathrm{zu} 40 \%$ in extranodalen Lokalisationen, und zwar hier am häufigsten im Gastrointestinaltrakt, im zentralen Nervensystem (ZNS), in der Haut und auch in der Lunge, jedoch nur ganz selten in der Prostata auftritt [1].

Die klinischen Symptome des malignen Lymphoms der Prostata sind vielfältig. Am häufigsten beschrieben ist die Obstruktion der unteren Harnwege bis zur Harnretention, was die Differenzierung von anderen Erkrankungen der Prostata sehr schwierig gestaltet $[10,12,13]$. In weiteren Studien wurden Symptome wie Hämaturie [14], beidseitiger Flankenschmerz und Müdigkeit [15], Dysurie [16] und auch Rückenschmerzen, wie in unserem Fall, beschrieben [9].

Eine Assoziation des Lymphoms wird außerdem mit der Psoriasis gesehen, möglicherweise wie in unserem Fall [17-19].

Die High-Grade-Lymphome werden prinzipiell mit kurativem Anspruch behandelt. Das Standardverfahren bei High-Grade-NHL ist die systemische Chemotherapie, kombiniert mit intrathekaler Applikation (bei ZNS-Befall) und Nachbestrahlung (,,involved field“ 30-40 Gy, bei ,bulky disease“ oder Restlymphomen) [20, 21].

Bei unserem Patienten wurde eine Polychemotherapie mit Cyclophosphamid, Doxorubicin, Vincristin, Prednison und Rituximab (R-CHOP) inklusive einer intrathekalen Tripeltherapie aus Methotrexat, Cytosinarabinosid und Hydrocortison ohne Benzylalkohol in Abständen von 14 Tagen zur ZNS-Prophylaxe eingeleitet. Geplant waren acht Zyklen
R-CHOP und sechs intrathekale Therapien. Die Verabreichung der intrathekalen Zytostatika wurde lumbal auf Höhe L4/L5, mit genügend Abstand zur vorbeschriebenen epiduralen Raumforderung, vorgenommen.

Bei einem ersten Zwischenstaging nach vier Zyklen hatten sich die Lymphommanifestationen deutlich zurückgebildet (partielle Remission). Nach weiteren zwei Zyklen R-CHOP zeigte ein CT zwar noch ein kleines Weichteilplus in der Prostataloge, im anschließenden PET-CT war jedoch kein vitales Lymphomgewebe mehr nachweisbar. In diesem Sinne konnte von einer kompletten Remission ausgegangen werden. Die ursprünglich vorgesehenen weiteren Therapiezyklen lehnte der Patient aus nichtmedizinischen Gründen $\mathrm{ab}$. In die konsolidierende Bestrahlung (Rezidivprophylaxe) der Prostata und Samenblase mit insgesamt 40 Gy über 4 Wochen willigte der $\mathrm{Pa}$ tient aber ein. Bei kürzlich durchgeführter Kontrolle auf dem medizinisch-onkologischen Ambulatorium präsentierte sich der Patient in gutem Allgemeinzustand.

\section{Literatur}

1. Jaffe ES, Harris NL, Stein H, et al., eds. World Health Organization classification of tumours, pathology and genetics of tumours of haematopoietic and lymphoid tissues. Lyon: IARC, 2001.

2. A clinical evaluation of the International Lymphoma Study Group classification of non-Hodgkin's lymphoma. By the Non-Hodgkin's Lymphoma Project. The American Society of Haematology. Blood 1997;89:3909-18.

3. Coupland S. Lymphoma of the prostate. Trans Pat Soc London 1877;28:179.

4. Bouet R, Thwaites D, Harris BS, et al. Asymptomatic follicular lymphoma of the prostate discovered by abnormal digital rectal examination. J Urol 2004;171:795-6.

5. Iczkowski KA, Lopez-Beltran A, Sakr WA. Hematolymphoid tumors of prostate. In: Eble JN, Sauter $\mathrm{G}$, Epstein JL, et al, eds. Tumor of the urinary system and male genital organs. Lyon: IAR Press, 2004:212.

6. Sarris A, Dimopoulos M, Pugh W, et al. Primary lymphoma of the prostate: good outcome with doxorubicin-based combination chemotherapy. J Urol 1995;153:1852-4.
7. Tomaru U, Ishikura H, Kon S, et al. Primary lymphoma of the prostate with features of low grade B-cell lymphoma of mucosa associated lymphoid tissue: a rare cause of urinary obstruction. J Urol 1999;162:496-7.

8. Chu PG, Huang Q, Weiss LM. Incidental and concurrent malignant lymphomas discovered at the time of prostatectomy and prostate biopsy. Am J Surg Pathol 2005;29:693-9.

9. Bostwick DG, Iczkowski KA, Amin MB, et al. Malignant lymphoma involving the prostate: report of 62 cases. Cancer 1998;83:732-8.

10. Bostwick DG, Mann RB. Malignant lymphoma involving the prostate: a study of 13 cases. Cancer 1985;56:2932-8.

11. King LS, Cox TR. Lymphosarcoma of the prostate. Am J Pathol 1951;27:801-23.

12. Mermershtain W, Bernharroch D, Lavrenkov K, et al. Primary malignant lymphoma of the prostate: a report of three cases. Leuk Lymphoma 2001; 42:809-11.

13. Sarlis NJ, Knight RA, Sarlis I, et al. Primary non-Hodgkin lymphoma of the prostate gland. Int Urol Nephrol 1993;25:163-8.

14. Alvarez CA, Rodriguez BI, Perez LA. Primary diffuse large B-cell lymphoma of the prostate in a young patient. Int Braz J Urol 2006;32:64-5.

15. Claikens B, Oyen R, Goethuys H, et al. NonHodgkin's lymphoma of the prostate in a young male. Eur Radiol 1997;7:238-40.

16. Miyahare T, Oyabul Y, Hayashi T, et al. A case of malignant lymphoma of the prostate. Nippon Hinyokika Gakkai Zasshi 2005;96:644-6.

17. Gelfand JM, Shin DB, Neimann AL, et al. The risk of lymphoma in patients with psoriasis. J Invest Dermatol 2006;126:2194-220.

18. Gelfand JM, Berlin J, Voorhees A, et al. Lymphoma rates are low but increased in patients with psoriasis. Arch Dermatol 2003;139:1425-9.

19. Margolis D, Bilker W, Hennessy S, et al. The risk of malignancy associated with psoriasis. Arch Dermatol 2001;137:778-83.

20. Abrahamson JS, Shipp MA. Advances in the biology and therapy of diffuse large B-cell lymphoma: moving toward a molecularly targeted approach. Blood 2005;106:1164-74.

21. Coiffer B, Lepage E, Brière J, et al. CHOP chemotherapy plus rituximab compared with $\mathrm{CHOP}$ alone in elderly patients with diffuse large B-cell lymphoma. N Engl J Med 2002;346:235-42.

\section{Korrespondenzanschrift}

Priv.-Doz. Dr. Uwe Schiemann

Klinik und Poliklinik

für Allgemeine Innere Medizin

Inselspital Bern (Universitätsspital)

Freiburgstraße

3010 Bern

Schweiz

Telefon (+41/31) 63-22111

Fax -24814

E-Mail: uwe-schiemann@insel.ch 